\title{
Modelling Trends of Cd4 Counts for Patients on Antiretroviral Therapy (Art) : After a Change in Who Guidelines in Kenya
}

\section{Caroline W Mugo ( $\square$ cwmugo@jkuat.ac.ke )}

Jomo Kenyatta University of Agriculture and Technology

\section{Ziv Shkedy}

Hasselt University

\section{Samuel Mwalili}

Jomo Kenyatta University of Agriculture and Technology

\section{Roel Braekers}

Hasselt University

Dolphine Wandede

Kenya Medical Research Institute

Christina Mwachari

Kenya Medical Research Institute

\section{Research Article}

Keywords: Highly active antiretroviral therapy(HAART), HIV/AIDS, CD4

Posted Date: March 11th, 2021

DOl: https://doi.org/10.21203/rs.3.rs-256325/v1

License: (9) (i) This work is licensed under a Creative Commons Attribution 4.0 International License.

Read Full License 


\title{
RESEARCH
}

\section{Modelling Trends of CD4 counts for patients on Antiretroviral Therapy (ART) : After a change in WHO guidelines in Kenya}

\author{
Caroline W Mugo ${ }^{1,2 *}$ \\ , Ziv Shkedy ${ }^{2}$, Samuel Mwalili ${ }^{1}$, Roel Braekers ${ }^{2}$, Dolphine Wandede ${ }^{3}$ and Christina Mwachari ${ }^{3}$.
}

\footnotetext{
${ }^{*}$ Correspondence:

cwmugo@jkuat.ac.ke

${ }^{1}$ Department of Statistics and

Actuarial Science, Jomo Kenyatta

University of Agriculture and

Technology, P.O BOX 62000,

00200 Nairobi, Kenya

Full list of author information is

available at the end of the article
}

\begin{abstract}
Background

In resource-limited settings, changes in CD4 counts constitute an important component in patient monitoring and evaluation of treatment response as these patients do not have access to routine viral load testing. In this study, we quantified trends on CD4 counts in patients on highly active antiretroviral therapy (HAART) in a comprehensive health care clinic in Kenya between 2011 and 2017. We evaluated the rate of change in CD4 cell count in response to antiretroviral treatment. We further assessed factors that influenced time to treatment change focusing on baseline characteristics of the patients and different initial drug regimens used. The study involved 529 naive HIV patients that had at least two CD4 count measurements for the period. The relationship between CD4 cell count and time was modeled using a semi parametric mixed effects model while the Cox proportional hazards model was used to assess factors associated with the first regimen change.
\end{abstract}

\section{Results}

The results demonstrated that CD4 counts increased over time and these trends were similar regardless of the treatment regimen used. Males were less likely to have drug regimens switch (adjusted hazard ratio (aHR) 0.5101, 95\% Cl: 0.1906 -1.3647) compared to females. Tenoforvir (TDF) based regimens had a lower drug substitution (aHR 0.2796, 95\% Cl: 0.0961-0.8629) compared to Zinovudine (AZT).

\section{Conclusion}

The backbone used was found to be associated with regimen changes among the patients with fewer switches being observed, with the use of TDF when compared to AZT. There was however no significant difference between TDF and AZT in terms of the change in CD4 count over time.

Keywords: Highly active antiretroviral therapy(HAART); HIV/AIDS; CD4

\section{Background}

Human immunodeficiency virus (HIV) epidemic has become one of the greatest threats to human health and development. The number of persons living with HIV has risen to about 36 million with a high percentage of about $67 \%$ being in SubSaharan Africa [1]. According to [1] Kenya has one of the largest HIV epidemic in 
the world with a prevalence rate of $5.9 \%$.

Combating the epidemic requires strategies that reduce the new infections and improvement of the survival rates of those already infected. In recent years, highly active antiretroviral therapy (HAART) has become available to the patients with World Health orgnaization (WHO) guidelines recommending initiation of ART in all adults and adolescents with HIV [2]. The benefits of combined antiretroviral therapy are well documented in literature [3][4]. After initiation of antiretroviral therapy(ART) most patients experience a reduction in HIV viral load combined with an increase in CD4 cell count which reduces the risk of HIV related events and death. A strong predictor of the progression to Acquired Immunodeficiency Syndrome (AIDS) is the CD4+ T-cell (CD4) count typically reported as an absolute level or count of cells (expressed as cells per cubic millimeter of blood)[5]. Changes in CD4 count constitute an important component in patient monitoring and evaluation of treatment response as these patients do not have access to routine viral load testing. WHO recommends CD4 count monitoring every six months and viral load testing only when the capacity exists [6]. However, the measurements of CD4 in most developing countries is not on a regular basis.

Revision of WHO guidelines in 2010 brought changes in the management of HIV infected patients among them use of less toxic antiretroviral drugs in first line [2]. In line with these recommendations, antiretroviral therapy guidelines for Kenya in 2011 [7] suggested that first-line regimens for HIV naïve adults and adolescents consist of two nucleoside reverse trancriptase inhibitors (NRTIs) as "backbone" along with one non-nucleoside reverse-transcrptase inhibitor (NNRTI). In the guideline, Lamivudine (3TC) was combined with one of two NRTIs and one of two NNRTI. The NRTIs were Zidovidine (AZT) and tenofovir (TDF) while the NNRTIs included efavirenz (EFV) and nevirapine (NVP).

The success of HAART nonetheless critically depends on regular patient follow-up to the treatment during their lifetime. ART drug regimens are however changed due to various reasons which include but not limited to toxicity,co-morbidity,pregnancy and treatment failure [8][9]. These drug regimen modifications limit treatment options and introduce challenges such as monitoring and adherence difficulties among the patients. These modifications have also been associated with poor clinical outcomes[10]. It is therefore of utmost importance to check how the patients adhere to the routine and assess factors that are associated with the treatment modifications.

In this study we aim to quantify the CD4 cell count trends over time for patients on combined ART in one of the comprehensive health care clinics in Nairobi,Kenya and evaluate the rate of change in the CD4 count in response to antiretroviral treatment.We also evaluate whether the evolution is related to the baseline characteristics and treatment regimen of the patient. Further, we estimate time until first drug regimen change and establish if the changes are associated with the baseline characteristics of the patient.In section 2 we discuss the methods used, the results are then displayed in section 3 and finally a comprehensive discussion and conclusion is presented in section 4 . 


\section{Methods}

\section{Study participants}

The data used in this study was sourced from the Kenya Medical Research Institute (KEMRI). In the original study Ethical review committee(ERC) permission was obtained locally and internationally; the protocol was reviewed for human subject concerns and approved by the Kenya Medical Research Institute ERC and University of California San Francisco Committee on Human Research. The study involved HIV naïve patients attending one of the comprehensive health care clinics in Nairobi, Kenya for the period between September 2011 to 2017. In this study,we included only 529 patients who had at least two CD4 count measurements during the follow up period and whose drug regimen was recorded.Informed consent was obtained from all participants included in the study.Baseline characteristics of the patients at initiation of ART such as gender,age, WHO clinical stage are also included.All methods were performed in accordance with the relevant guidelines and regulations.

\section{Treatments}

According to [7], the forth edition of the ART Kenya guidelines released in December 2011 the recommended first-line regimens for naïve adults and adolescents consisted of two nucleoside reverse transcriptase inhibitors (NRTIs) as "backbone" along with one non-nucleoside reverse-transcriptase inhibitor (NNRTI). In the giudeline, Lamivudine (3TC) was combined with one of two NRTIs and one of two NNRTI options implying that we have four treatment combinations for first line HAART. The NRTIs were zidovudine(AZT) and tenofovir (TDF) while the NNRTIs included efavirenz (EFV) and nevirapine (NVP).

During the follow up period some of the patients changed regimens for different reasons. These changes in regimen involved either the backbone or NNRTI. Whilst the number of changes made by a patient may be more than one ,in this study we considered the first regimen change as our outcome of interest.

\section{Statistical Analysis}

Exploratory data analysis and descriptive statistics was carried out to give an insight into the data.Baseline categorical variables were cross tabulated to give the proportions in different categories and a Chi-square test performed to find out if any association existed among the variables.

Time to treatment change

The outcome of interest which was time to first treatment change was calculated by subtracting the date of treatment modification from the date of ART initiation.Patients were censored if treatment change was not observed until the last visit to the clinic. This was done for patients who were lost to follow up and for those still alive at the end of study. Suppose we let $T$ be a random variable representing failure time in our case time to regimen or treatment switch. The probability of failure time occurring at exactly time $t$ can be formulated as:

$$
f(t)=\lim _{h \rightarrow 0} \frac{P[t \leq T<t+h]}{h}
$$


The cumulative distribution of the random variable $T$ is as shown

$$
F(t)=P(T \leq t), t>0,
$$

with a survival function

$$
S(t)=P[T \geq t]=1-F\left(t^{-}\right)
$$

The hazard function

$$
\lambda(t)=\lim _{h \rightarrow 0} \frac{P[t \leq T<t+h \mid T \geq t]}{h}
$$

The time to treatment change was estimated using Kaplan-Meier estimator. The $\log$-rank(LR) test was used to compare between groups of baseline characteristics and initial treatment allocations. The statistic is given by

$$
L R=\frac{\sum_{i=1}^{k}\left(E_{i}-O_{i}\right)^{2}}{E_{i}}
$$

where $O_{i}$ is the observed number of failures and $E_{i}$ is the expected number of failures. Under then null hypothesis that the survival distribution are the same for the groups, then the log-rank test statistic has a chi-square distribution.

The Cox proportion hazards model [11] was used to identify the baseline characteristics that could be associated with first treatment or regimen change. The model was formulated as;

$$
h_{i}\left(t \mid x_{i}\right)=h_{0}(t) \exp X_{i} \beta
$$

where $h_{0}(t)$ is a baseline hazard function that describes the risk for individuals with $X_{i}=0$ and $\exp X_{i} \beta$ is the relative risk representing a proportionate increase or reduction in risk, associated with a set of characteristics $X_{i}$. The hazard ratio with $95 \%$ confidence interval was used to test statistical significant association between time to first treatment change and the patient's baseline characteristics.

\section{Analysis of CD4 cell count}

CD4 cell counts were log-transformed to meet the assumption about stability of the variance with increasing CD4 cell count. Individual trajectory plots were obtained to give an indication of how the patient's CD4 count evolved over time. We constructed a mean profile of the log transformed CD4 over time in months. Further, the average profile plots were fitted for different baseline characteristics.

Let $y_{i j}$ denote the response for subject $i$ measured at occasion $j$. Further if we let $\mathbf{y}_{i}$ denote a vector of all repeated measurements for subject $i$ then we can formulate the general linear mixed effects model as:

$$
\mathbf{y}_{i}=X_{i} \beta_{i}+Z_{i} \mathbf{b}_{i}+\epsilon_{i}
$$


where $\mathbf{y}_{i}, \mathrm{i}=1,2,3 \ldots, n_{i}$ is a $n_{i}$-dimensional vector of the log transformed CD4 counts for patient $i$ at time $j, X_{i}$ and $Z_{i}$ are $n_{i} \times p$ and $n_{i} \times q$ matrices of known covariates, $\beta$ is a p-dimensional vector of fixed effects and $\mathbf{b}$ q-dimensional vector of subject specific random effects and $\epsilon_{i}$ is the residual component .

A simple parametric model may be adequate to describe subject-specific profiles in terms of random effects. However, the relevance of normality assumption on random effects may be questionable. Furthermore, the individual profiles are nonlinear making parametric models too restrictive. We propose a data-driven approach based on semi-parametric regression models used by [12]. In this approach, a patient specific random intercept is used to capture the correlation of CD4 cell count measurements over time within the patients. We assume patient specific random parameters for both linear and quadratic time effects to capture different evolution patterns between the patients. This is formulated as in the model below.

$$
y_{i}\left(t_{i}\right)=S\left(t_{i}\right)+b_{0 i}+b_{1 i} t_{i}+b_{2 i} t_{i}^{2}+\epsilon_{i} t_{i}
$$

Where $S\left(t_{i}\right)$ is a nonparametric component of the model and $b_{0 i}, b_{1 i}$ and $b_{2 i}$ are the patient specific random effects. The model allows smoothing with respect to time. First order derivative for each treatment groups were obtained and plotted with $95 \%$ confidence band to determine the effect of treatments on the rate of change in the logarithm of CD4 cell count over time.

\section{Results}

\section{Baseline characteristics}

A total of 529 patients with at least two measurements of CD4 count were included in the study. Table 1 provides a summary of the CD 4 count measurements and age of the patients.Subjects were followed up for a maximum of 2149 days. The median time of follow up was 208.5 days. The number of CD4 count measurements per subject ranged from two to fifteen with a median of 4 measurements. A majority of the patients $(55.6 \%)$ had CD4 cell count of less than 200 cells $/ \mathrm{mm}^{3}$ which was the previous cutoff point to start ART. The highest CD4 count being 1631 with a median value of 395 . The number of visits to the clinic ranged from two to fifty with a median of twenty two.

Categorical baseline characteristics are summarized in table 2. Most patients in the study were females at $65.2 \%$. At initiation of ART $62.2 \%$ of the patients were at WHO clinical staging stage II.

With regards to the drug regimen, a majority of the patients were on Lamivudine + Tenofovir + Efavirenz (64.1\%). Efavirenz was the most used among the NNRTI at $69.6 \%$ while Tenofovir was the most used NNRT at $69.2 \%$ as shown in table 3 . The individual profiles of the subjects are presented in Fig. 1. From the profiles we observe that there is within and between subject variability. The subjects start at different baseline CD4 counts and evolve differently over time. There is an indication that the overall trend is not linear over time. Initially most patient's CD4 count increases rapidly then stabilizes. 


\section{Time to treatment change}

The number of patients that had at least one treatment change account for about $10 \%$ of the patients. Log-rank test was used to test the difference between categories of baseline covariates with the probability of treatment modification. This test revealed the presence of significant difference among the categories of baseline NNRTI, NRTI, gender and WHO clinical stages. The Kaplan-Meir curves are shown in Fig. 2. The survival curve for time to treatment change shows steady increase on overall.

The cox regression analysis results are presented in table 4. Adjusting for the baseline characteristics we find that only the backbone was associated with the drug regimen changes $(\mathrm{aHR}=0.2796(95 \%$ C.I:0.0961-0.8629) $)$ which was also observed in the log-rank test.Patients initiated on NVP were at a higher risk of changing treatment compared to those on EFV.In addition, males were less likely to have treatment modification compared to females.

\section{Modelling CD4 cell count}

The logarithm CD4 count trend is the same for both NNRTI treatments as seen in Fig. 3. After initiation to ART the rise of logarithm CD4 count in patients on Efavirenz is faster compared to those on Nevaripine. At later time points we observe that the CD4 cell count of those taking EFavirenz is higher than for those on Nevirapine. However, there appears to be no difference between the trend for TDF and AZT. The fitted individual profiles for the patients and overall average trend of logarithm CD4 count from semiparametric model is shown in Fig. 4. There was a rise in the logarithm CD4 cell count in the first days after initiation of ART and thereafter it stabilizes.

The first order derivative of the semiparametric mixed model fitted allows estimation of the rate of change in CD4 counts.A derivative equal to zero implies a constant trend with respect to time. The Fig. 5 presents the rate of CD 4 change over time with the $95 \%$ confidence band. It was observed that the rate decreases to zero in the first days after initiation to ART on average and thereafter remains close to zero. However, a closer look on the individual profiles indicated that not all subjects got to zero.The confidence band was wide towards the end of study.

The model also allows comparison between different groups. The treatment response for the two NNRTI drugs looks the same for both EFV and NVP in the evolution of logarithm CD4 cell counts as shown in Fig. 6A and the rate of change Fig. 6B in the left panel and right panel respectively. Fig. 7 displays the difference in estimated rate of change between NVP and EFV.The $95 \%$ confidence band covers zero throughout the study an indication that there was no difference in the evolution of CD4 cell counts for patients taking either of the treatments. The trend of logarithm CD4 cell counts was estimated for the backbones TDF and AZT. The trends rise steadily for both NRTIs over time with differences observed at initiation of ART. AZT had higher logarithm CD4 cell counts over time compared to TDF as seen in Fig. 8A. A wider confidence band was observed towards the end of study with regard to the rate of change Fig. 8 B. A further investigation on the difference between estimated curves by the backbones AZT and TDF, showed that there were differences at initiation of treatment but thereafter there were no observable differences as seen in Fig. 9. 


\section{Discussion}

Analysis of the CD4 count is an important component in monitoring and evaluating progression of HIV in resource limited settings. This study aimed at describing the evolution of CD4 cell counts and the evaluating time to first treatment change among patients after initiation of ART.Majority of patients in the study were in WHO clinical stage II.The number of female patients was higher compared to males which could be explained by the fact that some patients were referrals from the Antenatal care (ANC) clinics. The evolution of CD4 count increases nonlinearly over time with rapid increase in CD4 cell count observed immediately after initiation of ART which then stabilizes with time. The change in CD4 count rises fast in the first 450 days of ART initiation. This was longer than in the study conducted in Ethiopia [12] where the rapid increase was observed in the first ten months. The trend of CD4 cell count over time was the same regardless of the NNRTI treatment given to the patients.

In line with previous studies, majority of the females were observed to have changed their treatment compared to the males [9][8]. From this study though males were less likely to have their drug regimens changed (adjusted hazard ratio 0.7944, 95\% CI: 0.67-0.95) compared to the females, this was not significant. This agrees with a study by [13] which showed no significant differences in treatment modification time by gender and regimen. Most of the changes were mainly from EFV to NVP which could be explained by the fact that EFV is not recommended for women who are pregnant.

On comparing the backbone,zinovudine based regimens had a higher drug substitution (aHR 2.067, 95\% CI: 1.81-2.36) compared to Tenofovir.This concurs with a study conducted by [14] in Ethiopia which concluded that TDF based regimens have more efficacy that AZT based regimens. Another study in Kenya [15] shows that TDF had lower modifications of the ART treatments.Further, a study in South Africa [16] showed that TDF seemed to perform better notably with less drug substitution. The trend in CD4 recovery for TDF and AZT seem to be similar with little difference observed at the initiation of ART.

A comparison of Efavirenz and Nevirapine in the evolution of the CD4 count indicated no differences. This is consistent with findings of in the study by [17] who found no significant differences among the study groups in the proportions with the increases in CD4-positive cells. Other studies conducted to compare the treatments have however indicated that EFV is better than NVP. For instance, studies by [18] showed that patients on EFV recovered more CD4 cell counts than those on NVP while [19] concluded that EFV-containing antiretroviral regimens were associated with superior clinical outcome, as measured by time to treatment failure. We have compared different baseline characteristics and drug regimens on time to first treatment substitutions and the rate of change in CD4 cell count over time.

Our study however had several limitations which include the fact that patients attending the comprehensive health care clinic were mainly referrals from antenatal care clinics and voluntary counseling centers and therefore may not completely represent all the HIV patients in Kenya. CD4 cell count measurement was not performed at particular time points and the number of measurements differ from the 
different patients. In addition, the analysis of drug regimen substitution was restricted to first treatment change only. Taking into account all the drug changes may reveal a different finding.

Ethics approval and consent to participate

Ethical review committee permission was obtained for the original study and approved by the Kenya Medical Research institute Ethical Review Committee and University of California San Francisco Committee on Human Research.Informed consent was obtained from all participants included in the study.

\section{Consent for publication}

Not applicable.

Availability of data and materials

The datasets during and/or analysed during the current study available from the corresponding author on reasonable request to be considered by the lead investigator.

Competing interests

The authors declare that they have no competing interests.

Funding

This study has not received funding. However, student research support was provided by VLIR-UOS under TEAM project "Capacity building network in biostatistics for public health innovation in Kenya".

\section{Author's contributions}

All the authors made substantial intellectual contributions to the study. Caroline $\mathrm{M}$ :Conceptualization, Data organization and analysis,writing of first draft manuscript, review of manuscript, final correction and review of manuscript.

Ziv S. Conceptualization, guidance in analysis of data, review of first manuscript, review and edit of final manuscript. Samuel M. Conceptualization, review of first draft manuscript,review and edit of final manuscript.

Roel B. Conceptualization, review of first draft manuscript, review of final manuscript.

Dolphine W. Data collection, data management, review of final manuscript.

Christina M. Lead investigator Nairobi cite, Design of study, data collection, review of first draft manuscript, review of final manuscript.

\section{Acknowledgements}

The authors acknowledge Director, Kenya Medical Research Institute (KEMRI), for authorizing this study and permitting the publication of this manuscript. We also acknowledge VLIR-UOS for student financial support to carry out the research under TEAM projects "Capacity building network in biostatistics for public health innovation in Kenya".

Author details

${ }^{1}$ Department of Statistics and Actuarial Science, Jomo Kenyatta University of Agriculture and Technology, P.O BOX 62000, 00200 Nairobi, Kenya. ${ }^{2}$ CENSTAT, Universitiet Hasselt, Agoralaan, 3590 Diepenbeek, Belgium. ${ }^{3}$ Kenya Medical Research Institute, P.O BOX 54840, 00200 Nairobi, Kenya.

References

1. UNAIDS: UNAIDS Gap Report, (2016)

2. WHO: Antiretroviral Therapy for HIV Infection in Adults and Adolescents: Recommendations for a Public Health Approach-2010 Revision. Geneva: World Health Organization, ??? (2010)

3. Mocroft, A., Ledergerber, B., Katlama, C., Kirk, O., Reiss, d. P, Monforte, A.d., Knysz, B., Dietrich, M., Phillips, A., Lundgren, J.D., et al.: Decline in the aids and death rates in the eurosida study: an observational study. The Lancet 362(9377), 22-29 (2003)

4. Palella Jr, F.J., Baker, R.K., Moorman, A.C., Chmiel, J.S., Wood, K.C., Brooks, J.T., Holmberg, S.D., Investigators, H.O.S., et al.: Mortality in the highly active antiretroviral therapy era: changing causes of death and disease in the hiv outpatient study. JAIDS Journal of Acquired Immune Deficiency Syndromes 43(1), 27-34 (2006)

5. Hoffman, J., Johan van, G., Colebunders, R., McKellar, M.: Role of the cd4 count in hiv management. HIV Therapy 4, 27-39 (2010)

6. WHO: Antiretroviral Therapy for HIV Infection in Infants and Children: Towards Universal Access. In HIV/AIDS Programme: Strengthening Health Services to Fight HIV/AIDS. World Health Organization, ??? (2010)

7. (NASCOP)., N.A.C.P.: Guidelines for Antiretroviral Therapy in Kenya. vol. 4th Edition., (2011)

8. Jima, Y.T., Angamo, M.T., Wabe, N.T.: Causes for antiretroviral regimen change among hiv/aids patients in addis ababa, ethiopia. Tanzania journal of health research 15(1) (2013)

9. Dimala, C.A., Bechem, N.N., Aroke, D., Kadia, B.M.: Motives for change of first-line antiretroviral therapy regimens in an unselected cohort of hiv/aids patients at a major referral centre in south-west cameroon. BMC research notes $10(1), 623(2017)$

10. Park, W.B., Choe, P.G., Kim, S.-H., Jo, J.H., Bang, J.H., Kim, H.B., Kim, N.J., Oh, M.-D., Choe, K.W.: Early modification of initial haart regimen associated with poor clinical outcome in hiv patients. AIDS Research and Human Retroviruses 23(6), 794-800 (2007)

11. Cox, D.: Regression models and life tables (with discussion). Journal of the Royal Statistical Society.Series B (Methodological) 34(2), 187-220 (1972) 
12. Awoke, T., Worku, A., Kebede, Y., Kasim, A., Birlie, B., Braekers, R., Zuma, K., Shkedy, Z.: Modeling outcomes of first-line antiretroviral therapy and rate of cd4 counts change among a cohort of hiv/aids patients in ethiopia: A retrospective cohort study. PLoS ONE 11(12) (2016)

13. Shenoy, S., Shrivastava, D., Malalur, C., Varma, M., Kunder, S.K., Arivazhahan, A., Saravu, K.: Modification of first-line antiretroviral therapy in treatment-naive, hiv positive patients. Journal of Clinical \& Diagnostic Research 11(10) (2017)

14. Ayele, T., Jarso, H., Mamo, G.: Immunological outcomes of tenofovir versus zidovudine-based regimens among people living with hiv/aids: a two years retrospective cohort study. AIDS research and therapy 14(1), 5 (2017)

15. Owuor, A., Lule, G., Otieno, C., Omonge, E., Maritim, M., Memiah, P.: Modification of antiretroviral therapy in a cohort study of hiv-infected patients attending an urban teaching hospital in kenya. Int J Virol AIDS 1, 003 (2014)

16. Velen, K., Lewis, J.J., Charalambous, S., Grant, A.D., Churchyard, G.J., Hoffmann, C.J.: Comparison of tenofovir, zidovudine, or stavudine as part of first-line antiretroviral therapy in a resource-limited-setting: a cohort study. PloS one 8(5), 64459 (2013)

17. Van Leth, F., Phanuphak, P., Ruxrungtham, K., Baraldi, E., Miller, S., Gazzard, B., Cahn, P., Lalloo, U., Van Der Westhuizen, I., Malan, D., et al.: Comparison of first-line antiretroviral therapy with regimens including nevirapine, efavirenz, or both drugs, plus stavudine and lamivudine: a randomised open-label trial, the $2 \mathrm{nn}$ study. The lancet 363(9417), 1253-1263 (2004)

18. Cozzi-Lepri, A., Phillips, A.N., d'Arminio Monforte, A., Piersantelli, N., Orani, A., Petrosillo, N., Leoncini, F., Scerbo, A., Tundo, P., Abrescia, N., et al.: Virologic and immunologic response to regimens containing nevirapine or efavirenz in combination with 2 nucleoside analogues in the italian cohort naive antiretrovirals (i. co. na) study. The Journal of infectious diseases 185(8), 1062-1069 (2002)

19. Philip, K., Naiel, N., Clinton, W., Glenda, K., Sylvia, M.: Comparison of nevirapine- and efavirenz-containing antiretroviral regimens in antiretroviral-naive patients: A cohort study. HIV Clinical Trials 3(4), 296-303 (2002)

Figures

Figure 1 Individual Profiles of logarithm CD4 cell count over time.

Figure 2 Kaplan- Meir curves for time to treatment change by different baseline characteristics.(a)Overall,(b) By gender,(c) By NRTI (d) By NNRT

Figure 3 Graph of logarithm CD4 count over time in days.(a) By backbone, (b) By NNRT treatments

Figure 4 Fitted individual CD4 count profiles with average smoothed line over time.

Figure 5 Estimated rate of change of logarithm CD4 cell count over time.

Figure 6 A.Predicted logarithm CD4 and B.Estimated rate of change of log CD4 over time by NNRTI.

Figure 7 Estimated difference in predicted log CD4 cell count and rate of change in CD4 cell count over time between EFV and NVP. 
Figure 8 (A)Predicted logarithm CD4 and (B) Estimated rate of change of logarithm CD4 cell count over time by backbone.

Figure 9 Estimated difference between the predicted log CD4 cell count over time by NRTI backbone.

Table 1 Summary continuous Characteristics

\begin{tabular}{|c|l|r|c|c|}
\hline Variable & Minimum & Maximum & Median & Mean \\
\hline Age & 18 & 70 & 42 & 42.66 \\
\hline CD4 & 1 & 1631 & 395 & 400.6 \\
\hline Log CD4 & 0 & 7.39 & 5.97 & 5.77 \\
\hline Observation time in days & 0 & 2149 & 208.5 & 341.9 \\
\hline No of visits per patient & 1 & 50 & 22 & 22.1 \\
\hline No of CD4 measurements & 2 & 15 & & \\
\hline \hline
\end{tabular}

Table 2 Summary of Categorical Baseline Characteristics

\begin{tabular}{|c|l|r|c|}
\hline Variable & Categories & Count & Percentage(\%) \\
\hline Gender & Female & 345 & 65.2 \\
\hline & Male & 184 & 34.8 \\
\hline WHO stage & Stage 1 & 76 & 14.4 \\
\hline & Stage 2 & 329 & 62.2 \\
\hline & Stage 3 & 73 & 13.8 \\
\hline & Stage 4 & 11 & 2.0 \\
\hline & Unknown & 41 & 7.8 \\
\hline \hline
\end{tabular}

Table 3 Treatments at baseline

\begin{tabular}{|l|l|r|}
\hline Backbone & EFV(\%) & NVP(\%) \\
\hline 3TC TDF & $339(64.1)$ & $27(5.1)$ \\
\hline 3TC AZT & $29(5.5)$ & $134(25.3)$ \\
\hline \hline
\end{tabular}

Table 4 Cox-regression analysis of factors associated with time to treatment change.

\begin{tabular}{|c|l|r|c|c|}
\hline Variable & Categories & UHR $(95 \% \mathrm{Cl})$ & $\mathrm{AHR}(95 \% \mathrm{Cl})$ & $\mathrm{p}$-value \\
\hline Gender & Female & 1 & 1 & \\
\hline & Male & $0.3435(0.1322-0.8923)$ & $0.5101(0.1906-1.3647)$ & 0.1800 \\
\hline NNRTI & Efavirenz & 1 & 1 & \\
\hline & Nevirapine & $4.629(2.048-10.46)$ & $1.6984(0.5677-5.0806)$ & 0.3434 \\
\hline Backbone & AZT & 1 & 1 & \\
\hline & TDF & $0.1817(0.0775-0.4258)$ & $0.2796(0.09061-0.8629)$ & 0.0267 \\
\hline
\end{tabular}


Figures

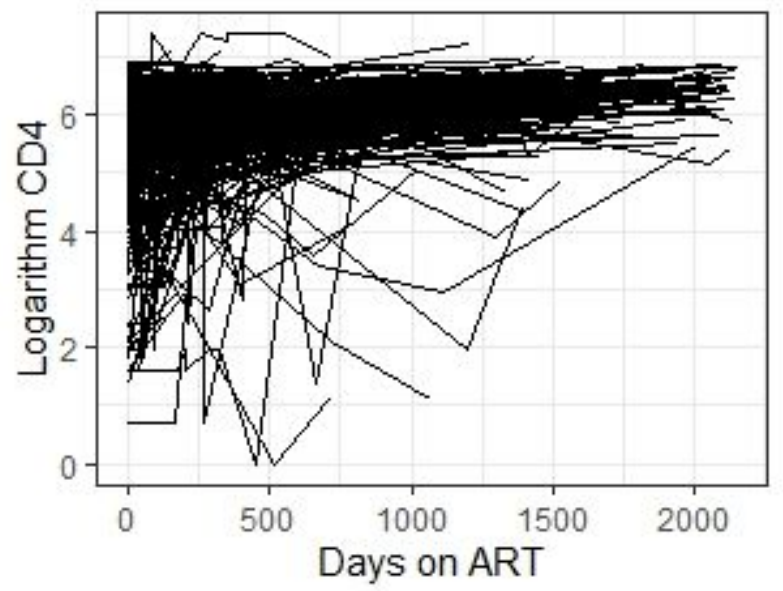

Figure 1

Individual Profiles of logarithm CD4 cell count over time.

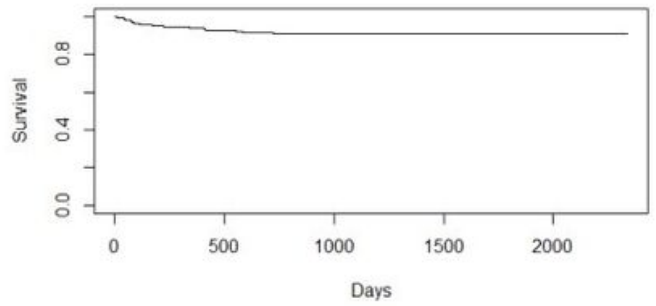

(a) Overall

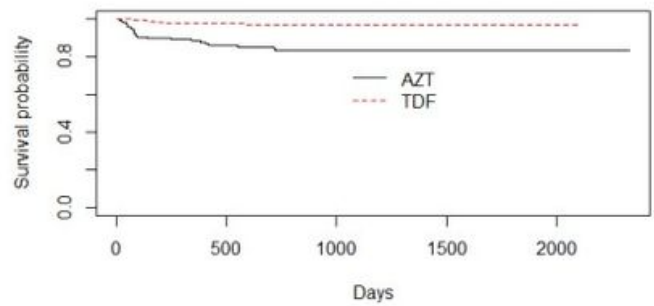

(c) By NRTI

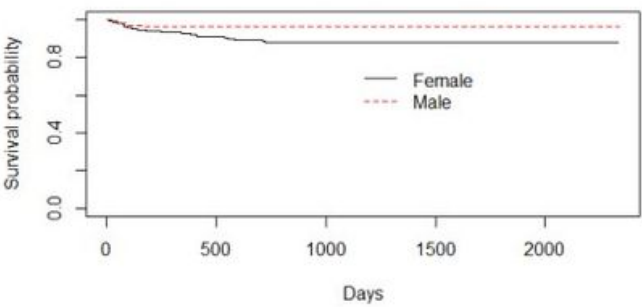

(b) By Gender

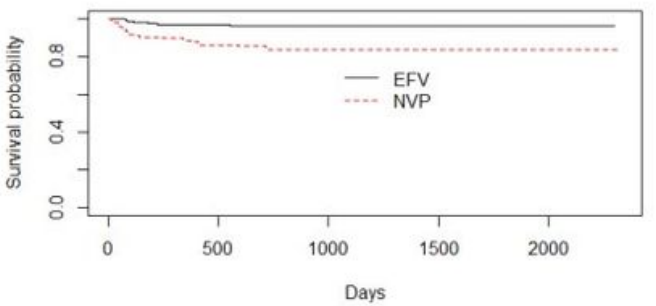

(d) By NNRT

Figure 2 
Kaplan- Meir curves for time to treatment change by different baseline characteristics.(a)Overall,(b) By gender,(c) By NRTI (d) By NNRT.

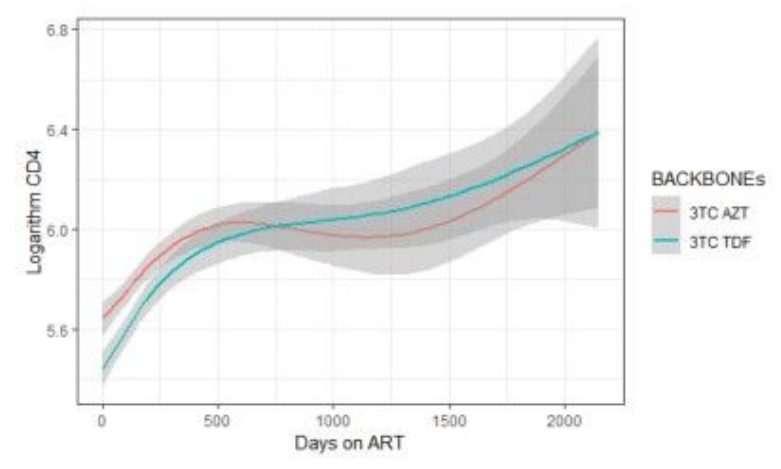

(a) By backbone

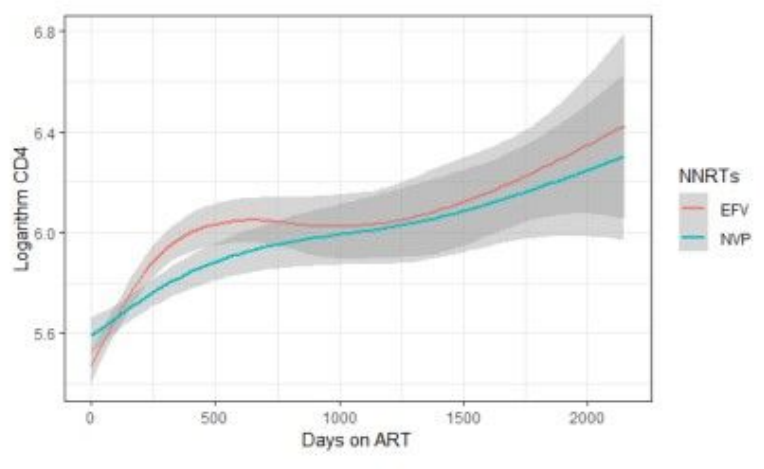

(b) By NNRT

\section{Figure 3}

Graph of logarithm CD4 count over time in days.(a) By backbone, (b) By NNRT treatments

\section{Smooth logarithm CD4}

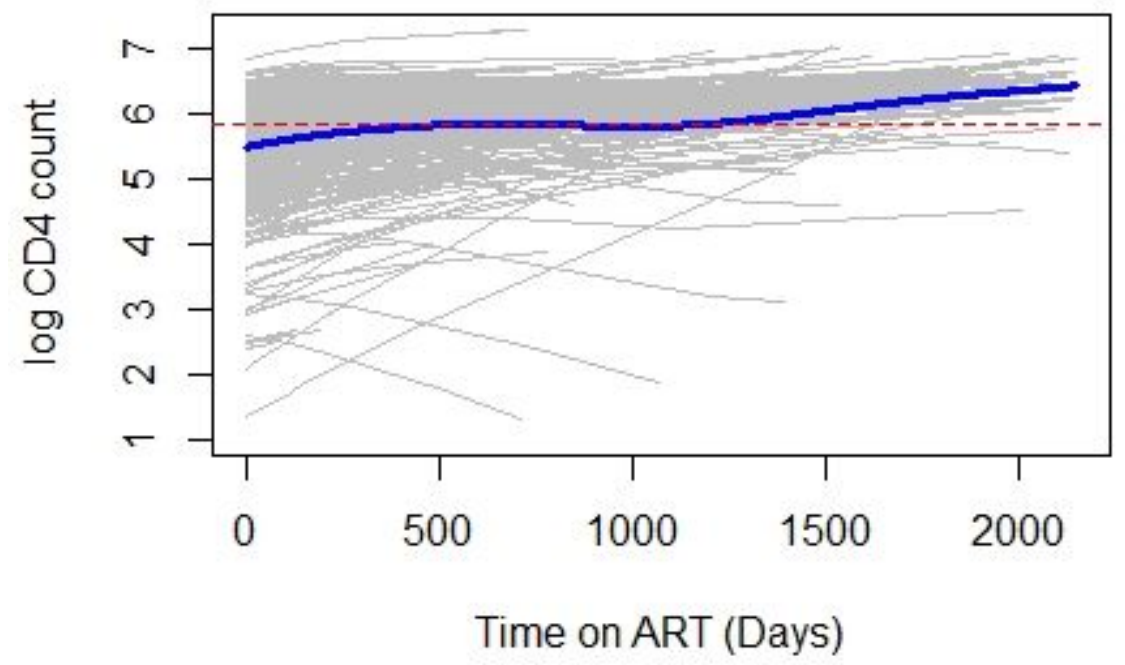

Figure 4

Fitted individual CD4 count profiles with average smoothed line over time. 


\section{Overall rate}

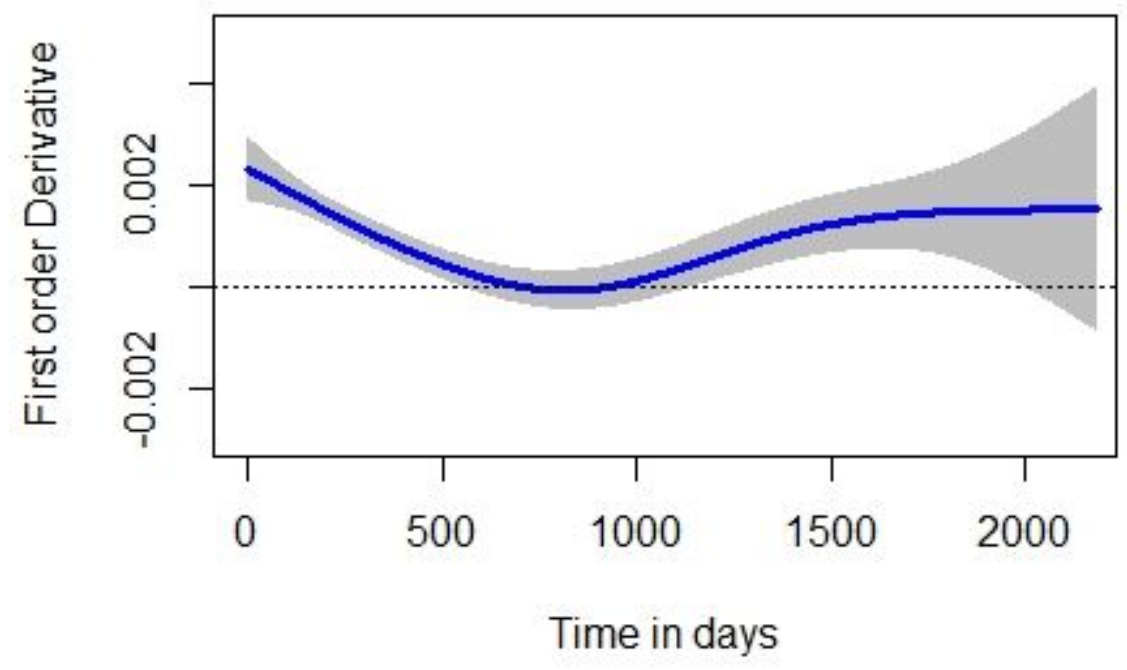

Figure 5

Estimated rate of change of logarithm CD4 cell count over time.

A

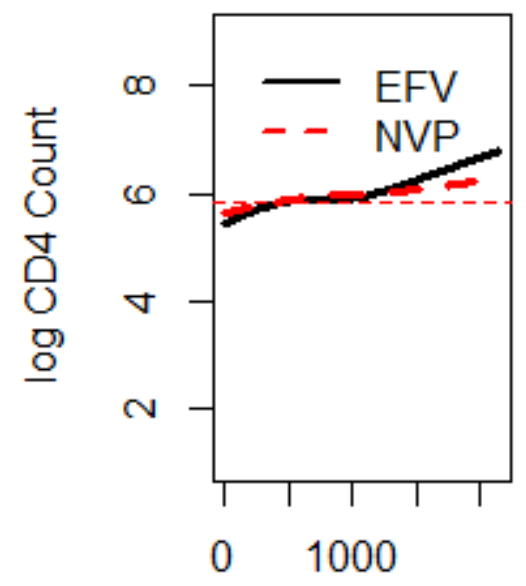

Time on ART (days)
B

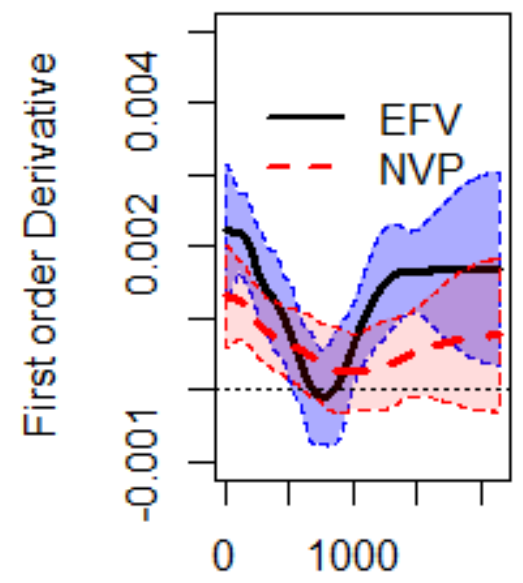

Time on ART (days)

Figure 6

A.Predicted logarithm CD4 and B.Estimated rate of change of log CD4 over time by NNRTI. 


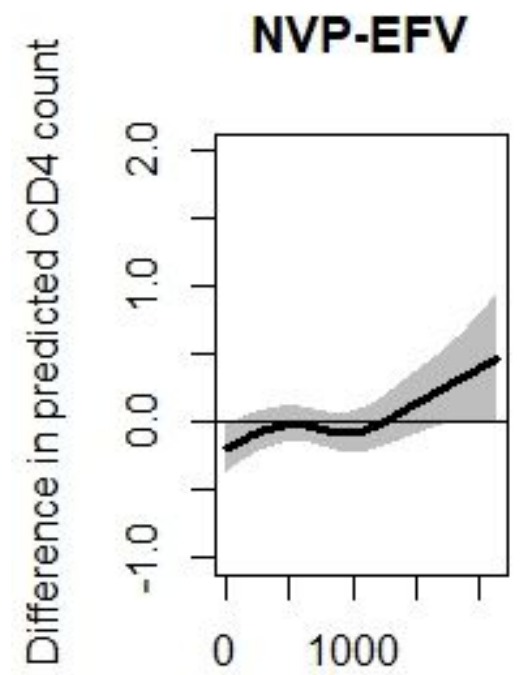

Time on ART (days)

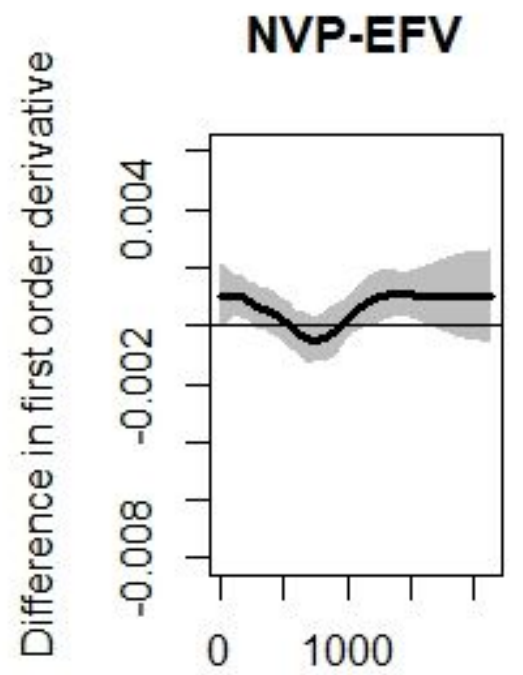

Time on ART (Days)

Figure 7

Estimated difference in predicted log CD4 cell count and rate of change in CD 4 cell count over time between EFV and NVP.

A

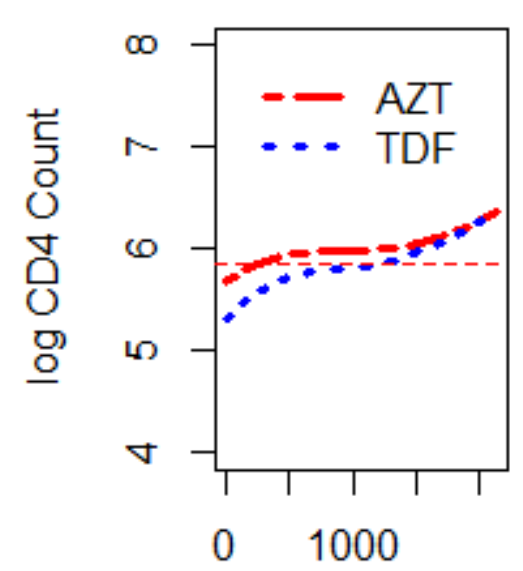

Time on ART (days)
B

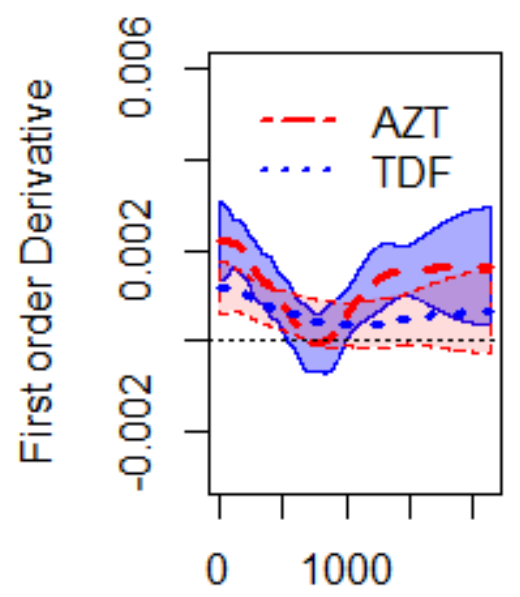

Time on ART (days)

Figure 8

(A)Predicted logarithm CD4 and (B) Estimated rate of change of logarithm CD4 cell count over time by backbone. 

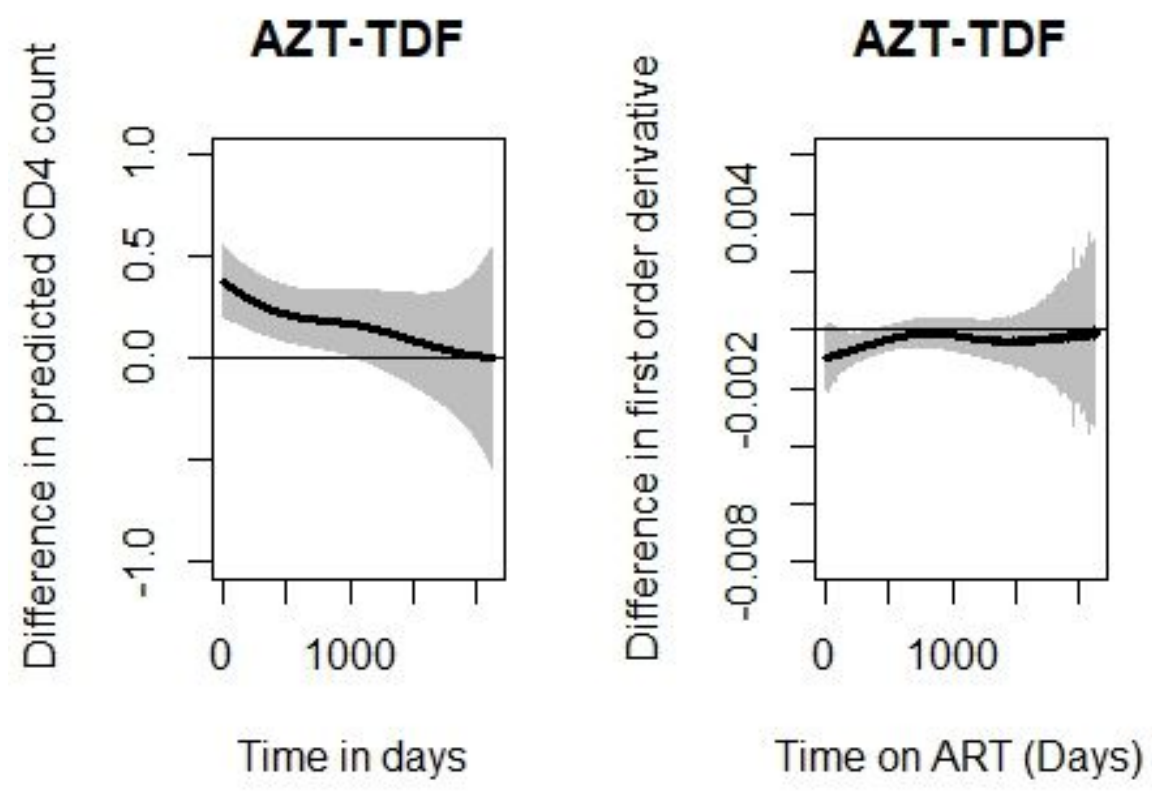

Figure 9

Estimated difference between the predicted log CD 4 cell count over time by NRTI backbone. 